\title{
Zur Rechtewahrnehmung von Autoren und Verlegern innerhalb der VG Wort
} Landgericht München I, Teil-Urteil vom 24. Mai 2012 - 7 O 28640/11

\section{Ein pauschaler Abzug von den Vergütungen, die auf die Autoren entfallen, zugunsten ihrer Verleger aufgrund der Verteilungspläne der VG Wort stellt einen Verstoß gegen das in § 7 UrhWG niedergelegte Willkürverbot dar. (Leitsatz der Redaktion)}

\section{Teil-Urteil}

- I. Es wird festgestellt, dass die Beklagte seit dem Jahr 2008 nicht berechtigt ist, bei ihrer jährlichen Ausschüttung den auf verlegte Werke des Klägers entfallenen Vergütungsanteil unter Berücksichtigung folgender Abzüge zu berechnen:

Abzug eines Verlegeranteils gemäß $\S 3$ Abs. 1 bis 3 der Verteilungspläne Wissenschaft der Beklagten in der im Zeitpunkt der jeweiligen Ausschüttung jüngsten Fassung, zuletzt idF vom 21. Mai 2011;

Abzüge, die sich aus $\S 12$ der Verteilungspläne Wissenschaft der Beklagten in der im Zeitpunkt der jeweiligen Ausschüttung jüngsten Fassung, zuletzt idF vom 21. Mai 2011 ergeben.

II. Im Übrigen wird die Klage abgewiesen.

\section{Tatbestand}

Die Beklagte ist eine Verwertungsgesellschaft und der Kläger ist deren Mitglied und Bezugsberechtigter. Er begehrt die Feststellung, dass die Verteilung der Einnahmen durch die Beklagte unrechtmäßig erfolgt, zudem begehrt er detaillierte Auskunft, welche Abzüge von seinen verlegten Werken gemacht wurden und kündigt im Wege der Stufenklage die Geltendmachung der Zahlung von weitergehenden Ausschüttungen an.

Die Beklagte ist eine Verwertungsgesellschaft. Sie verwaltet Zweitverwertungsrechte, vorrangig die Vergütungsansprüche nach $\S 44$ a ff. UrhG. Neben den Urhebern sind auch Verleger Mitglieder der Beklagten. Beide Personenkreise bilden je drei der sechs Berufsgruppen nach § 2 Absatz 2 der Satzung. Die Klägerin verfügt über eine Satzung und Verteilungspläne, welche jeweils von der Mitgliederversammlung im Mehrheitsverfahren beschlossen wurden.

In der Satzung der Beklagten in der Fassung vom 22. Mai 2010, genehmigt am 11. Oktober 2010 (K 4 b) findet sich folgende Regelung:

$$
\text { „\$9/1 }
$$

Nr. 1. Soweit mit angemessenen Mitteln feststellbar, hat jeder Berechtigte den auf die Nutzung seines Werkes entfallenen Anteil am Ertrag zu erhalten.
Nr. 3 Den Verlegern steht ein ihrer verlegerischen Leistung entsprechender Anteil am Ertrag der VG WORT zu."

In dem Verteilungsplan Wissenschaft - Fassung 21. Mai 2011 (K 5) - findet sich in I $33 \mathrm{Nr}$. 1 folgende Regelung:

\section{„§ 3 Grundsätze der Verteilung}

Die Verteilungssummen bestehen zur gleichen Hälfte aus einem Urheber- und einem Verlagsanteil. Beide Teile werden den Berechtigten gegenüber gesondert abgerechnet und verteilt."

In I § $3 \mathrm{Nr}$. 2 und 3 des Verteilungsplans sind Änderungen des Verteilungsverhältnisses zwischen Urheber und Verleger enthalten. Im für die Verleger ungünstigsten Fall stehen diesen $38 \%$ zu.

Die Beklagte sieht eine jährliche pauschale Zahlung an bestimmte Berufsverbände vor. In $\S 12$ des Verteilungsplans Wissenschaft lautet es unter der Überschrift

\section{„Ausschüttungen an Urheberorganisationen:}

Nach Ablauf von 4 Jahren ab dem Jahr, für das Rückstellungen gemäß § 1 Abs. 3 gebildet wurden, werden die für noch nicht wahrnehmungsberechtigte Urheber zurückgestellten Mittel an diejenigen Urheberorganisationen ausgeschüttet, denen Berechtigte ihre Ansprüche übertragen haben und welche die VG WORT entsprechend freistellen.

\section{Zur Zeit sind folgende Organisationen anerkannt:}

\section{Deutscher Hochschulverband}

Gesellschaft Deutscher Chemiker

Deutsche Physikalische Gesellschaft

Über die Berücksichtigung weiterer Organisationen beschließt der Verwaltungsrat gemäß § 9 Abs. 7 der Satzung der ehemaligen VG Wissenschaft GmbH.

$$
\text { (...)." }
$$


Der Kläger ist Autor wissenschaftlicher und nichtfiktionaler Werke. Am 26. Januar 1984 hat er einen Wahrnehmungsvertrag mit der Beklagten abgeschlossen (K 1). In diesem Wahrnehmungsvertrag lautet es unter anderem:

\section{„\$1. Der Verwertungsgesellschaft Wort werden aus allen} dem Vertragsschließenden zustehenden oder erwachsenden Urheber- und Verlagsrechten ( $v g l$. § 2) folgende Rechtsbefugnisse zur treuhänderischen Wahrnehmung eingeräumt: (...)

§3. Satzung und Verteilungsplan, auch soweit sie künftig geändert werden sollten, bilden einen Bestandteil des Vertrags. Beschließt die Mitgliederversammlung in Zukunft Abänderungen des Wahrnehmungsvertrags, so gilt auch diese Abänderung als Bestandteil dieses Vertrages."

Seit dem 13. Mai 1994 ist er Mitglied der Beklagten. In der Folgezeit hat der Beklagte Verträge mit Verlagen geschlossen, die eine Abtretung der Zweitverwertungsrechte vorsahen [...].

Seit dem 01. Januar 2008 hat der Beklagte die in Anlage K 2 aufgeführten zehn Werke gemeldet. Dabei handelt es sich nach Angaben des Klägers bis auf das Werk "Auf Kosten der Künstler“, welches im Jahr 2004 in dem Autorenblatt „Die Feder" erschienen ist und am 22. März 2010 gemeldet wurde, ausschließlich um verlegte Werke.

Die Beklagte hat an den Kläger im Jahr 2008 191,75€, im Jahr $200960 €$, im Jahr 2010 1.854,19€ und im Jahr 2011960 $€$ ausgeschüttet. Der Kläger ist der Ansicht, dass in der Abrechnung auch unverlegte Werke des Klägers wie etwa Zeitungsartikel berücksichtigt sein müssten. Die Abrechnung vom 19. März 2010 über 1.854,19 € liegt vor als K 7. Es handelt sich um eine pauschalisierte Abrechnung. Eine spezifizierte Abrechnung bietet die Beklagte in $§ 4$ der Verteilungspläne Wissenschaft (K 5) gegen Zahlung einer Gebühr von $15 €$ an. § 4 lautet:

„Berechtigte können gegen Erstattung einer Verwaltungsgebühr in Höhe von $€ 15$,- einen detaillierten Abrechnungsauszug oder eine Steuerbescheinigung von der VG WORT erhalten."

Der Kläger ist der Ansicht, dass die Beteiligung der Verleger an den auf sein Werk entfallenden Ausschüttungsanteil zu Unrecht erfolgt. Er habe durch den mit der Beklagten geschlossenen Wahrnehmungsvertrag aus dem Jahr 1984 alle zum damaligen Zeitpunkte bestehenden und alle zukünftigen Vergütungsansprüche, welche auf der Schrankenregelung der §§ 44a UrhG basieren, im Voraus abgetreten. Er hat deshalb keine Rechte mehr gehabt, die er an die Verleger seiner Werke hätte abtreten können.

Die Ausschüttung an die in $\S 12$ der Verteilungspläne Wissenschaft genannten Berufsverbände sei willkürlich und zu Unrecht erfolgt. Obwohl diese Ausschüttungen nicht aus dem regelmäßig zu verteilenden Aufkommen stammen, sondern aus aufzulösenden Rückstellungen sei der Kläger dadurch zu Unrecht belastet.

Der Wahrnehmungsvertrag sei ein Geschäftsbesorgungsvertrag nach § 675 BGB und der Kläger habe gegen die Beklagte einen Herausgabeanspruch auf alles Erlangte, abzüglich aller Verwaltungskosten und der gesetzlich zulässigen Aufwendungen für kulturelle und soziale Zwecke nach §§ 7, 8 UrhWG. Der Kläger müsse sich nur solche Ausgaben anrechnen lassen, die an andere Berechtigte gehen. Verleger und Berufsverbände seien aber keine anderen Berechtigten.

Die vorprozessual vertretene Auffassung der Beklagten, dass der Kläger mit Abschluss des Wahrnehmungsvertrages die Satzung und die Verteilungspläne der Beklagten in ihren jeweils aktualisierten Fassungen als Teil der Vertragsbeziehungen anerkannt habe, sei unzutreffend, da es sich um rechtswidrige Abzüge handele und solchen nicht zugestimmt werden könne.

Die Vorschriften § 3 Abs. 1 bis 3 der Verteilungspläne Wissenschaft und § 9 Absatz 1 Ziffer 3 der Satzung - jedenfalls in der seit 2008 geltenden Fassung - würden gegen § 63 a UrhG und das AGB-Recht verstoßen. Nach der revidierten Fassung des $\S 63$ a UrhG kann der Urheber seine Vergütungsansprüche zwar im Voraus an einen Verleger abtreten, sofern dieser sie zur gemeinsamen Wahrnehmung in eine Verwertungsgesellschaft einbringt. Daraus folge aber keine Befugnis, auch solche Verleger an der Verteilung zu beteiligen, die gar keine Rechte eingebracht haben. Die Verabschiedung der Verteilungspläne habe mithin gegen den Treuhandgrundsatz verstoßen.

Wahrnehmungsverträge unterliegen den AGB-Regeln. Durch die Vorausabtretungen, die in allen Wahrnehmungsverträgen enthalten seien, seien Verleger daran gehindert, Rechte zu erwerben. Weiter gebe es eine Inhaltskontrolle anhand der Anforderungen des UrhWG und des § 242 BGB, selbst wenn die Satzungsbestimmungen mehrheitlich beschlossen wurden.

Soweit in der Satzung des Deutschen Hochschulverbandes eine Abtretung vorhanden ist, sei nicht klar, ob es sich dabei um eine Abtretung von Vergütungs- oder von Auszahlungsansprüchen handele. Vergütungsansprüche könne der Urheber seit dem Inkrafttreten des § 63 a UrhG am 01. Juli 2002 nicht mehr abtreten und eine Abtretung von Auszahlungsansprüchen scheitere bereits daran, dass sie nicht auf eindeutig nachgewiesenen Rechten der Verbände beruhe.

Der Kläger begehrt Feststellung, dass die von der Beklagten gemachten Abzüge zu Unrecht erfolgt sind, begehrt Auskunft und kündigt im Wege der Stufenklage die Geltendmachung eines Schadensersatzanspruchs an.

Der Kläger beantragt, 
Es wird festgestellt, dass die Beklagte seit dem Jahr 2008 nicht berechtigt ist, bei ihrer jährlichen Ausschüttung der auf verlegte Werke des Klägers entfallenden Vergütungsanteile diese unter Berücksichtigung folgender Abzüge zu berechnen:

Abzug eines Verlegeranteils gemäß $§ 3$ Abs. 1 bis 3 der Verteilungspläne Wissenschaft der Beklagten in der im Zeitpunkt der jeweiligen Ausschüttung jüngsten Fassung, zuletzt idF vom 21. Mai 2011;

Abzüge, die sich aus § 12 der Verteilungspläne Wissenschaft der Beklagten in der im Zeitpunkt der jeweiligen Ausschüttung jüngsten Fassung, zuletzt idF vom 21. Mai 2011 ergeben.

\section{$[\ldots]$}

[...]

Die Beklagte beantragt,

die Klage abzuweisen.

Die Beklagte erhebt die Einrede der Verjährung. Beiträge 4, 5, 6 und 10 (Liste K 2) seien bereits verjährt. Auch der Beitrag in dem Autorenblatt "Die Feder" sei ein verlegtes Werk. Der Beitrag Nr. 9 der Liste K2 sei noch nicht gemeldet.

Der Beklagte ist der Ansicht, dass die Verleger an den gesetzlichen Vergütungsansprüchen zu beteiligen seien. Diese Vergütungsansprüche bestünden seit der Urheberrechtsreform von 1965. Von Anbeginn an sei die Verteilung durch Verwertungsgesellschaften erfolgt und stets sei eine Beteiligung der Verleger vorgesehen gewesen. Urheber und Verleger seien eine Symbiose, da nur eine gemeinsame Verwertung erfolgreich sein könne. Lediglich die Höhe der Beteiligung habe geschwankt. Da es sich um ein Masseverfahren handele, müsse typisiert und pauschaliert werden. Eine genauere Abrechnung würde einen unangemessenen Verwaltungsaufwand bedeuten.

Die Bedeutung der Verleger zeige sich auch darin, dass der Autor bei der Werkanmeldung Angaben zum Verlag machen muss, in dem sein Werk erschienen ist. Ohne eine Publikation durch einen Verlag könne im Printbereich nicht davon ausgegangen werden, dass das Werk im hinreichenden Maße verbreitet wird. Selbst wenn die Abtretung an den Verleger zeitlich nach dem Abschluss eines Wahrnehmungsvertrages mit der Beklagten erfolge, so soll der Verlag nicht von der Verteilung ausgeschlossen werden. Dies zeige sich auch in den standardmäBig verwendeten Autorenverträgen [...], in denen immer auch eine Abtretung der Verwertungsrechte zugunsten der Verlage vorgesehen sei.
Der Kläger sei an den Verteilungsplan gebunden. Dieser sei mit Zustimmung aller Berufsgruppen der Beklagten zustande gekommen. Bei dem Verhältnis zwischen den Parteien handele es sich um eine Geschäftsbesorgung nach Maßgabe der Satzung und des Verteilungsplans.

Bei Neufassung des $\S 63$ a UrhG zum 01. Januar 2008 sei nicht gewollt gewesen, dass eine Beteiligung der Verleger entfalle. Laut der amtlichen Begründung habe die Aufnahme des $\S 63$ a Satz 2 UrhG dafür sorgen sollen, dass die Ansprüche der Verleger nicht leer laufen, sondern erhalten bleiben. Der Gesetzgeber habe in der amtlichen Begründung [...] ausdrücklich ausgeführt, ",dass die Verleger auch in Zukunft an den Erträgen der VG Wort angemessen zu beteiligen seien."

Das AGB-Recht sei für den vorliegenden Fall nicht anwendbar und selbst wenn es anwendbar wäre, so würde keine unangemessene Benachteiligung des Klägers vorliegen. Verleger würden eine vergleichbare Leistung zur Vermittlung urheberrechtlich geschützter Werke erbringen, wie es bei Veranstaltern, Tonträgerherstellern, Sendeunternehmen, Filmherstellern und Datenbankherstellern der Fall ist, für die das Gesetz ausdrücklich ein Leistungsschutzrecht vorsehe. Bis zur Einführung des $\S 63$ a UrhG habe auch der Kläger eine Beteiligung der Verlage akzeptiert.

In Bezug auf die Beteiligung der Urheberorganisationen ist die Beklagte der Ansicht, dass deren Beteiligung gemäß § 12 der Verteilungspläne historisch bedingt sei und aus der Zeit rühre, als die beiden Verwertungsgesellschaften VG Wort und VG Wissenschaft im Jahr 1978 fusionierten. Ihre Beteiligung würde zumindest bis in das Jahr 1981 zurückreichen.

Die Urheberorganisationen hätten bis 2008 jährlich Ausschüttungen in Höhe von genau 281.211 $€$ erhalten. Dieser Betrag entspreche 550.000 DM und sei seit 1991 unverändert gewesen. Dann sei der Verein Deutscher Ingenieure aus der Verteilung ausgeschieden und der bislang auf diesen Verband entfallende 14-prozentige Anteil sei mit Wirkung ab dem Ausschüttungsjahr 2009 nicht mehr zur Verteilung gelangt, so dass nur noch $241.841 €$ zur Ausschüttung gelangten.

Die Ausschüttungen nach § 12 der Verteilungspläne würden Beiträge der Mitglieder, die keine rechtzeitige Meldung vorgenommen haben, betreffen. Es handele sich um eine Verteilung von Rückstellungen. Zudem seien die Beträge verhältnismäßig gering und würden sich auf die Zahlungen an den Kläger lediglich in geringer Höhe auswirken. Im Verhältnis zur gesamten Ausschüttungssumme handele es sich im Jahr 2008 um 1,75\%, im Jahr 2009 um 1,18 \% und im Jahr 2010 um 0,59\%.

Darüber hinaus würden die Ausschüttungen an Urheberorganisationen nach einem mit dem Deutschen Patent- und Markenamt als der Aufsichtsbehörde genau abgestimmten Verfahren vorgenommen. Die genaue Vorgehensweise sei in einem an 
das DPMA gerichteten Schreiben vom 05. August 2010 erläutert (B 9) und laufe bei den drei Organisationen wie nachfolgend dargestellt ab:

Der Deutsche Hochschulverband und die Gesellschaft Deutscher Chemiker haben Regelungen in ihre Satzungen aufgenommen, welche einen Übergang von Auszahlungsansprüchen seiner Mitglieder gegen die Beklagte enthalten.

In § 10 der Satzung der Gesellschaft Deutscher Chemiker (B 11) ist folgende Regelung enthalten:

\section{„Mitgliedsbeiträge}

\section{[... ] Die von der Verwertungsgesellschaft Wort gemäß deren Verteilungsplan auf die Mitglieder der GDCh entfal- lenen Ausschüttungsbeiträge sind, soweit die Mitglieder dieser Beiträge nicht selbst bei der Verwertungsgesellschaft Wort durch rechtzeitige Anmeldung ihrer Werke in Empfang nehmen, zusätzliche, der GDCh unmittelbar zustehende Mit- gliedsbeiträge."}

Die Deutsche Physikalische Gesellschaft (DPG) hat keine entsprechende Satzungsregelung. Nach einem mit der Aufsichtsbehörde abgestimmten Verfahren schreibt diese Gesellschaft eine bestimmte Anzahl von Mitgliedern, die keine Ansprüche gegen die Klägerin geltend gemacht haben, an und lässt sich von diesen deren Vergütungsansprüche in einem Formular (B 12) abtreten.

Die Beklagte hat sich mit der Aufsichtsbehörde auf ein vereinfachtes Nachweisverfahren geeinigt. Die Urheberorganisationen müssen belegen, dass eine bestimmte Zahl von Mitgliedern keine Ansprüche geltend gemacht hat, bzw. eine Abtretung vorgenommen hat. Beim Deutschen Hochschulverband sind es 479 Mitglieder, bei der Gesellschaft Deutscher Chemiker 56 Mitglieder und bei der Deutschen Physikalischen Gesellschaft 218 Mitglieder.

Weiter ist die Beklagte der Ansicht, dass der Kläger mit Abschluss seines Wahrnehmungsvertrages akzeptiert habe, dass die Verteilungspläne und deren künftige Änderungen, die von der Mitgliederversammlung beschlossen werden, Bestandteil des Vertrages sind und sich die Abrechnung und Auszahlung der Vergütungen nach Satzungen und Verteilungsplänen richtet. Auch die Regeln zur Verjährung seien ihm bekannt.

Autoren seien im Vergleich zu Verlagen meistens die Schwächeren. Deshalb würden zahlreiche Autorenverträge eine umfassende Rechteabtretung an die Verlage vorsehen. Die Verteilungspläne der Verwertungsgesellschaften hätten deshalb den Vorteil, dass sie eine angemessene Beteiligung in § 7 UrhWG garantieren würden. Um den Urhebern diesen Anteil zu garantieren, seien schon lange vor Einführung des § 63 a UrhG manche Vergütungsansprüche verwertungsgesellschaftspflichtig ausgestaltet worden.
Bei Neufassung des $\S 63$ a UrhG sei nicht gewollt gewesen, dass eine Beteiligung der Verleger entfallen solle. Laut der amtlichen Begründung habe die Aufnahme des § 63 a Satz 2 UrhG dafür sorgen sollen, dass die Ansprüche der Verleger nicht leer laufen, sondern erhalten bleiben.

Verleger würden eine vergleichbare Leistung zur Vermittlung urheberrechtlich geschützter Werke erbringen, wie es bei Veranstaltern, Tonträgerherstellern, Sendeunternehmen, Filmherstellern und Datenbankherstellern der Fall ist, für die das Gesetz ausdrücklich ein Leistungsschutzrecht vorsehe. Bis zur Einführung des $\S 63$ a UrhG habe auch der Kläger eine Beteiligung der Verlage akzeptiert.

Daraufhin erwidert der Kläger, dass die angemeldeten Ansprüche nicht verjährt seien. Die Beklagte habe eine unzutreffende Verjährungsberechnung vorgenommen. Maßgeblich für den Verjährungsbeginn sei nicht die Beschlussfassung des Verwaltungsrates der Beklagten, sondern der Zeitpunkt, zu dem die Beklagte eine Abrechnung über Ausschüttungen zu dieser Publikation vornimmt.

Der von der Beklagten vertretene Ansatz der Theorie der statuarischen Verteilungspläne könne nicht mehr vertreten werden. Die Rechtsprechung zur Inhaltskontrolle der Satzungen von Monopolvereinen und zur Anwendung des AGB-Gesetzes und die Entscheidung des EuGH vom 09. Februar 2012 „Luksan" (K 8) würden diesem Verständnis deutlich entgegenstehen.

Darauf erwidert die Beklagte, dass insbesondere das EuGHUrteil "Luksan“ nicht entgegenstehe. Bei diesem Urteil sei es um die in Österreich praktizierte cessio legis des Filmurheberrechts gegangen. Es sei darum gegangen, ob Vergütungsansprüche statt den Filmurhebern den Filmproduzenten zustünden, obwohl letztere eigene Vergütungsansprüche haben. Während hinsichtlich der Verwertungsrechte eine Vermutung der Rechtseinräumung zu Gunsten der Filmproduzenten zulässig sei, soweit diese Vermutung widerlegbar bleibe, sei eine Vermutung der Abtretung des dem Hauptregisseur des Filmwerks zustehenden Anspruchs auf gerechten Ausgleich an den Produzenten dieses Werks nicht möglich. [...]

\section{Entscheidungsgründe}

Die Klage hat Erfolg, soweit der Kläger die Feststellung verlangt, dass der Abzug eines Verlegeranteils und die Ausschüttungen an Berufsvereinigungen gemäß § 12 der Verteilungspläne Wissenschaft zu Unrecht erfolgten.

\section{1.) Auslegung der Klageanträge}

Der Kläger begehrt mit Ziffer 1 seines Klageantrags Feststellung, dass die Verteilungspraxis der Beklagten in zwei Punkten rechtswidrig ist. 


\section{2.) Zulässigkeit der Klage}

Das Feststellungsinteresse für den Klageantrag ist gegeben. Das Erheben einer - an sich vorrangigen - Leistungsklage hätte den Nachteil, dass zukünftige Zeiträume nicht abgedeckt wären. Soweit die Beklagte vorgetragen hat, dass es an einem Rechtsschutzinteresse fehlen würde, weil sich die Beklagte auch an gerichtliche Feststellungen, die in einem Zahlungsverfahren einfließen würden, halten würde, kann sie nicht durchdringen. Es mag zwar sein, dass sich die Beklagte an Feststellungen in einem entsprechenden Urteil gebunden fühlen würde. Eine rechtliche Gewähr dafür gäbe es jedoch nicht.

\section{3.) Aktivlegitimation}

Der Kläger ist Mitglied der Beklagten und hat im nichtverjährten Zeitraum Werke angemeldet und Ausschüttungen erhalten. Insofern ist er unmittelbar durch das Verteilungsverfahren der Beklagten betroffen. Es kann insofern für die Beurteilung der derzeit zu beurteilenden Anträge dahinstehen, ob die Beklagte mit ihren Einwendungen gegen einzelne vom Kläger gemeldete Werke durchdringt, da der Kläger unstreitig Werke im streitgegenständlichen Zeitraum angemeldet hat.

\section{4.) Prüfungsmaßstab}

Nach § 7 Satz 1 UrhWG haben Verwertungsgesellschaften die Einnahmen aus ihrer Tätigkeit nach festen Regeln so zu verteilen, dass ein willkürliches Vorgehen bei der Verteilung ausgeschlossen ist. Da die Verwertungsgesellschaften eine Vielzahl von Ansprüchen verwalten müssen, sind Typisierungen, Pauschalisierungen und Schätzungen zulässig und im gewissen Umfang steht ihnen ein billiges Ermessen bei der Verteilung zu (Schulze in: Dreier/ Schulze, 3. Aufl., § 7 UrhWG, Rn. 6). Allerdings muss beachtet werden, dass es sich trotz kollektiver Wahrnehmung um individuelle Rechte handelt. Deshalb hat eine individuelle Verteilung der eingezogenen Vergütungen Vorrang, solang sie mit zumutbarem Aufwand möglich ist (Schulze in: Dreier/ Schulze, 3. Aufl., § 7 UrhWG, Rn. 7).

\section{5.) Pauschale Beteiligung der Verleger}

Verlage haben kein eigenes Leistungsschutzrecht. Deshalb können sie allenfalls vom Urheber abgeleitete Rechte bei der Beklagten beibringen. Im Falle des Klägers führt die in dem Wahrnehmungsvertrag aus dem Jahr 1984 erklärte Abtretung aller bestehenden und zukünftigen Rechte an die Beklagte dazu, dass die Verlage, die Werke des Klägers verlegen, von ihm keine Rechte erwerben können. Soweit ersichtlich, handelt es sich um keinen Einzelfall, da zumindest eine Vielzahl von Autoren einen solchen Wahrnehmungsvertrag unterzeichnen, um in den Genuss der Ausschüttungen der Beklagten zu kommen. In dieser Art von Fallgestaltungen werden Abtretungen an Verlage deshalb regelmäßig zeitlich später liegen. Sie gehen dann ins Leere, weil der jeweilige Autor keine Rechte mehr hat, die er abtreten kann.
Die Beklagte hat nach § 3 ihrer Verteilungspläne von den Vergütungen, die auf den Kläger entfallen, einen pauschalen Abzug zugunsten der Verleger gemacht. Dieser Abzug stellt einen Verstoß gegen das in § 7 UrhWG niedergelegte Willkürverbot dar. Denn der Verleger des Klägers hat kein eigenes Nutzungsrecht und konnte auch vom Kläger kein Nutzungsrecht durch Abtretung erwerben. Denn der Kläger hat bereits im Jahr 1984 alle Nutzungsrechte der streitgegenständlichen Art an die Beklagte abgetreten. Insofern ist § 63 a UrhG nicht einschlägig. Es ist mithin keine Rechtsgrundlage ersichtlich, weshalb der Verleger des Klägers an den Ausschüttungen, die auf den Veröffentlichungen des Klägers herrühren, beteiligt werden soll.

Etwas anderes folgt auch nicht daraus, dass die Verteilungspläne der Beklagten bereits seit Beitritt des Klägers eine Ausschüttung an die Verleger vorsehen und dass der jeweils gültige Verteilungsplan durch einen Mehrheitsbeschluss der Mitgliederversammlung beschlossen wurde. Der Kläger als einzelner Autor hat faktisch keine andere Möglichkeit, als die Verteilungspläne der Beklagten zu akzeptieren, wenn er Anteil an den auf $\S 44$ a ff. UrhG basierenden Ausschüttungen haben möchte. Es liegt ein faktisches Monopol vor, da gemäß dem gesetzlichen Leitbild stets nur eine Verwertungsgesellschaft zur Wahrnehmung der Ansprüche berufen ist. Insofern kann aus dem Beitritt des Klägers zur Beklagten kein konkludenter Rechtsverzicht hergeleitet werden. Etwas anderes folgt auch nicht aus der Tatsache, dass die Beklagte den Verteilungsplan in der Mitgliederversammlung verabschiedet hat. Es widerspricht dem Willkürverbot in § 7 UrhWG und ist daher auch nicht durch Mehrheitsbescheid zu begründen, wenn Anteile der auf das Werk des Klägers entfallenden Vergütung an den nicht berechtigten Verleger ausbezahlt werden.

Wie oben dargelegt, können bei der Berechnung der Ausschüttungen Pauschalisierungen erforderlich sein, um eine bessere Handhabung der Ausschüttungen zu gewährleisten. Allerdings ist es einzelnen Autoren nicht zumutbar, wenn sie durch die Pauschalisierungen offensichtlich unangemessen benachteiligt werden. Dies ist vorliegend der Fall. Selbst wenn es im Ergebnis nämlich so wäre, dass den Verlegern in Bezug auf die Gesamtausschüttungen der Beklagten etwa $50 \%$ zustehen würden, da $50 \%$ der Autoren für ihre Werke keine Vorausabtretungen an die Beklagte vorgenommen hätten, so kann doch nicht übersehen werden, dass Verleger an dem individuellen Werk des Klägers keine Rechte haben. Deshalb wäre das Verteilungssystem für den Kläger offensichtlich ungerecht und er würde mit seinem Werk die Abtretungen anderer Autoren finanzieren, die ihrerseits zu Unrecht begünstigt wären, soweit sie nach altem Recht alle Rechte an die Verleger abgetreten haben, bevor die Werke bei der Beklagten gemeldet wurden. Da der Kläger in den Jahren 2008 bis 2011 insgesamt Ausschüttungen in einer GröBenordnung von etwa $3.000 €$ erhalten hat, handelt es sich auch nicht um Bagatellbeträge, die zugunsten einer vereinfachten Handhabung hingenommen werden müssten. 
Die Beklagte verteidigt ihre Verteilungspraxis mit unterschiedlichsten Argumenten. Zum einen wird vorgetragen, dass die Beteiligung der Verleger historisch gewachsen ist. Zum anderen wird vorgetragen, dass die Berücksichtigung des genauen auf einen Urheber und auf seinen Verlag entfallenen Anteils organisatorisch nicht umsetzbar sei.

Soweit die Beklagte der Meinung ist, dass die Beteiligung der Verleger aus historischen Gründen gerechtfertigt sei, da eine Verwertung eines Werkes nur dann sinnvoll möglich sei, wenn Urheber und Verleger zusammen arbeiten, kann sie damit nicht durchdringen. Der Gesetzgeber hat dem Verleger kein eigenes Leistungsschutzrecht eingeräumt und es ist nicht Aufgabe einer Verwertungsgesellschaft, eine Billigkeitsgesichtspunkten entsprechende Umverteilung contra legem vorzunehmen. Es mag dahinstehen, welche Intentionen der Gesetzgeber bei der Änderung des $\S 63$ a UrhG zum 01. Januar 2008 hatte. Nach der neuen Fassung kann ein Autor unter bestimmten Voraussetzungen Rechte an einen Verlag im Voraus abtreten. Dies betrifft aber nicht den Fall, dass er die Rechte vollständig an jemand anderes - hier die Beklagte - abtritt.

Soweit die Beklagte in der Klageerwiderung auf die amtliche Begründung der Neufassung des $§ 63$ a UrhG Bezug nimmt, und aus den Stellen

\section{„[...] Ansprüche dem Urheber erhalten bleiben und in der} Praxis nicht leer laufen [...]"

\section{„[...] Ein Ausschluss der Verleger von der pauschalen Ver-} gütung wäre angesichts der von ihnen erbrachten erheblichen Leistung auch sachlich nicht hinnehmbar [...]"

„Der neue Satz 2 soll gewährleisten, dass die Verleger auch in Zukunft an den Erträgen der VG Wort angemessen zu beteiligen sind. [...]"

herleiten möchte, dass der Gesetzgeber die Verteilungspraxis der Beklagten billigt, führt dies zu weit. Eine Gesetzesbegründung zur Neufassung des § 63 a UrhG kann allenfalls zur Auslegung dieser Vorschrift herangezogen werden und nicht zur Bestimmung des Begriffs „Willkür" in § 7 UrhWG verwendet werden, soweit es um Fälle geht, in denen die in $\S 63$ a UrhG geregelte Abtretung an den Verlag gar nicht erfolgte. Andernfalls wären die verfassungsrechtlich gebotenen Grenzen von Grundrechtseinschränkungen verlassen, wenn aus der Gesetzesbegründung zu § 63 a UrhG eine faktische hälftige Enteignung auch der Autoren hergeleitet würde, die Vorausabtretungen an die Beklagte vorgenommen haben.

Es ist nicht ersichtlich, dass es einen erheblichen organisatorischen Mehraufwand darstellen würde, wenn die genaue Rechteverteilung bei der Berechnung der Ausschüttung Berücksichtigung finden würde. Soweit sich die Beklagte auf diesen Mehraufwand beruft, fehlt es an substantiiertem Vortrag. Es mag sein, dass eine genaue Erfassung des Urheberanteils in früheren Jahrzehnten einen erheblichen Verwaltungsaufwand bedeutet hätte. Unter Berücksichtigung des heutigen Standes der Datenverarbeitung ist nicht ersichtlich, wo ein organisatorisches Problem liegen sollte, wenn die Beklagte tatsächlich an einer genauen Erfassung der Rechte interessiert wäre. Dem Sachvortrag der Beklagten fehlt es insofern an der erforderlichen Substantiiertheit.

Die Wertungen der "Luksan-Entscheidung" des EuGH haben keinen Einfluss auf den vorliegenden Fall, da es im vorliegenden Fall nicht um die Wirksamkeit der Abtretung von Rechten an einen Verlag geht. Der Kläger hat seine Rechte an die Beklagte als Verwertungsgesellschaft abgetreten. Ob die Entscheidung Auswirkungen auf andere von der Beklagten vertretenen Urheber hat, kann dahinstehen, da diese Entscheidung nicht vom beantragten Feststellungstenor umfasst ist.

\section{6.) Ausschüttung an Berufsorganisationen}

Wie oben dargelegt, ist die Beklagte als Verwertungsgesellschaft aus § 7 UrhWG gehalten, Ausschüttungen nur an Berechtigte vorzunehmen. Insofern ist eine pauschale Ausschüttung an Berufsverbände ohne den Nachweis, dass sie bestimmte Rechte abgetreten bekommen hat, unzulässig. Es ist nicht Aufgabe der Beklagten, diese Verbände finanziell zu unterstützen.

Allerdings muss es möglich sein, dass die Mitglieder dieser Verbände ihre Auszahlungsansprüche - für den Fall, dass sie diese Rechte nicht geltend machen - an den Verband abtreten. Eine solche Regelung verstößt nicht gegen $\S 63$ a UrhG, weil nicht auf "gesetzliche Vergütungsansprüche" verzichtet wird, sondern eine bedingte Abtretung von Zahlungsansprüchen erfolgt.

Für den streitgegenständlichen Zeitraum ist die Auszahlung als unzulässig anzusehen, weil die Beklagte nicht darlegen konnte, dass die tatsächlich an die Berufsverbände erfolgten Zahlungen in einem angemessenen Verhältnis zu den Vergütungsansprüchen stehen. Aus den vorgelegten Unterlagen ergibt sich, dass die Beklagte die Berechtigung anhand einer pauschalisierten Berechnungsweise vornimmt. Dabei legt sie den durchschnittlichen Ausschüttungsbetrag der angemeldeten Urheber zugrunde. Dieser Ansatz ist willkürlich.

Aus dem als B 9 vorliegenden Schreiben der Beklagten an die Aufsichtsbehörde ist das Vorgehen so beschrieben, dass der Deutsche Hochschulverband nachweisen muss, dass er 479 Mitglieder haben muss, die keine Ansprüche an die Beklagte angemeldet haben. Die Gesellschaft Deutscher Chemiker muss 56 Mitglieder nachweisen. Eine Prüfung, dass die so ermittelten Personen tatsächlich und in welchem Umfang publiziert haben, erfolgt nicht. Im Hinblick auf die Deutsche Physikalische Gesellschaft verlangt die Beklagte, dass 218 DPG-Mitglieder, die in dem jeweiligen Jahr publiziert haben, ohne hierfür individuelle Leistungen der VG WORT in Anspruch genommen zu haben, eine Abtretungserklärung vorlegen. Der genaue Umfang der 
Veröffentlichung wird nicht geprüft und es ist nicht ersichtlich, inwieweit tatsächlich Ansprüche an die Verbände übergegangen sind. Auch wenn eine Pauschalisierung grundsätzlich möglich ist, so hat die Möglichkeit der Pauschalisierung ihre Grenzen doch dort, wo ohne Bezug zum Tatsächlichen gehandelt wird.

Insofern müsste auf Grund echter (zufälliger oder sonst repräsentativer) Stichproben der Umfang der relevanten Veröffentlichungen ermittelt und auf den Umfang der demnach an den Verband abgetretenen Ansprüche aller Verbandsmitglieder hochgerechnet werden.
Die EuGH-Entscheidung "Luksan“ ist auch auf diese Problemstellung nicht anwendbar, da hier keine Nutzungsrechte an die Berufsverbände übergehen, sondern nur nicht geltend gemachte Auszahlungsansprüche. [...] (Entscheidung von der Redaktion bearbeitet.)

\section{Unrechtmäßigkeit einer Anhalteverfügung (§ 8 Abs. 2 Satz 1 KultGüRückG)} Verwaltungsgericht Köln, Urteil vom 25. April 2012 - 10 K 3537/11

1. Der zeitliche Anwendungsbereich für eine Anhalteverfügung gemä $§ 8$ Abs. 2 Kult GüRückG ist nur für solche Objekte eröffnet, die nach dem 26. April 2007 aus dem Hoheitsgebiet eines Vertragsstaates verbracht wurden.

2. Voraussetzung für die Rechtmäßigkeit einer Anhalteverfügung ist im Falle der Einleitung eines Bezeichnungsverfahrens gemäß § 6 Abs. 2 Satz 1 KultGüRückG die öffentliche Bekanntmachung desselben vor Erlass der Verfügung. (Leitsätze des Einsenders*)

\section{Tenor}

- Der Bescheid des Beklagten vom 17. Juni 2011 wird aufgehoben.

Der Beklagte trägt die Kosten des Verfahrens.

Das Urteil ist hinsichtlich der Kosten gegen Sicherheitsleistung in Höhe von $110 \%$ des jeweils zu vollstreckenden Betrages vorläufig vollstreckbar.

Die Berufung wird zugelassen.

\section{Tatbestand}

Die Klägerin betreibt unter der aus dem Rubrum ersichtlichen Bezeichnung in Köln ein Auktionshaus, das sich u.a. mit der Versteigerung von Kunstgegenständen, Möbeln und Porzellanen beschäftigt. Die Klägerin gibt für jede ihrer Auktionen vorher einen Katalog heraus, in dem sie die angebotenen Kunstgegenstände bildlich darstellt und beschreibt, einen Schätzpreis angibt und, soweit möglich, die Provenienz nennt.

Für den 18. Juni 2011 kündigte die Klägerin ihre Auktion 984 „Pre-Columbian Art" an. Auch für diese Auktion hatte sie einen Katalog herausgegeben.

Mit Verbalnote vom 17. Juni 2011 ersuchte die Botschaft von Mexiko in Berlin den Beklagten [das Land Nordrhein-Westfalen, Anmerkung der Redaktion] um Anordnung der Anhaltung der im Versteigerungskatalog der Klägerin mit den Katalognummern

Karl-Sax Feddersen, Syndikus des Kunsthauses Lempertz, Repräsentanz Berlin.
199 - 214, 216 - 217, 219 - 223 und 225 sowie 226 versehenen Artefakte. Vorausgegangen war eine Besichtigung der Gegenstände durch den mexikanischen Archäologen Jaime Alejandro Bautista Valdespino am 15. Juni 2011 und eine von ihm am 16. Juni 2011 abgegebene eidesstattliche Versicherung, wonach die Objekte mexikanischen Ursprungs seien. [...]

Mit Bescheid vom 17. Juni 2011 ordnete der Beklagte die Anhaltung der im Versteigerungskatalog der Klägerin mit den Katalognummern 199-214, 216-217, $219-223$ sowie $225-226$ versehenen Artefakte an. Er ordnete die sofortige Vollziehung der Anhaltung an.

Zur Begründung der Anhaltung führt er im Wesentlichen Folgendes an: Die Anhaltung habe nach § 8 Abs. 2 KultGüRückG zu erfolgen. Es bestehe der dringende Verdacht der unrechtmäßigen Ausführung der Gegenstände aus dem Gebiet der Vereinigten Mexikanischen Staaten. Die Objekte seien mexikanischen Ursprungs. Sie seien von mesoamerikanischen Kulturen aus dem heutigen mexikanischen Staatsgebiet vor der spanischen Eroberung hergestellt worden. Es handle sich demnach um besonders bedeutsames nationales Kulturgut der Vereinigten Mexikanischen Staaten. Die Eintragung in das "öffentliche Verzeichnis für archäologische und historische Denkmäler und Flächendenkmäler" (Registro Publico de Monumentos y Zonas Arqueologicos e Historicos), das im Internet unter der Adresse http://www.registropublico.inah.gob.mx zugänglich sei, werde auf der Grundlage des Gutachtens des Archäologen Jaime Alejandro Bautista Valdespino in Kürze erfolgen. Das förmliche Eintragungsverfahren sei mit der Entsendung des Archäologen eingeleitet worden. Die Artefakte seien unter Verstoß gegen die 